Draft VERsion SEPTEMBer 14, 2021

Preprint typeset using $\mathrm{LT}_{\mathrm{E}} \mathrm{X}$ style emulateapj v. 5/2/11

\title{
THE ENVIRONMENT OF BARRED GALAXIES IN THE LOW-REDSHIFT UNIVERSE
}

\author{
Ye Lin ${ }^{1}$, Bernardo Cervantes Sodi ${ }^{1,2},{\text { Cheng } \text { Li }^{1} \text {, Lixin WAng }}^{1}$, And Enci Wang ${ }^{1}$ \\ Draft version September 14, 2021
}

\begin{abstract}
We present a study of the environment of barred galaxies using a volume-limited sample of over 30,000 galaxies drawn from the Sloan Digital Sky Survey. We use four different statistics to quantify the environment: the projected two-point cross-correlation function, the background-subtracted number count of neighbor galaxies, the overdensity of the local environment, and the membership of our galaxies to galaxy groups to segregate central and satellite systems. For barred galaxies as a whole, we find a very weak difference in all the quantities compared to unbarred galaxies of the control sample. When we split our sample into early- and late-type galaxies, we see a weak but significant trend for early-type galaxies with a bar to be more strongly clustered on scales from a few $100 \mathrm{kpc}$ to $1 \mathrm{Mpc}$ when compared to unbarred early-type galaxies. This indicates that the presence of a bar in early-type galaxies depends on the location within their host dark matter halos. This is confirmed by the group catalog in the sense that for early-types, the fraction of central galaxies is smaller if they have a bar. For late-type galaxies, we find fewer neighbors within $\sim 50 \mathrm{kpc}$ around the barred galaxies when compared to unbarred galaxies form the control sample, suggesting that tidal forces from close companions suppress the formation/growth of bars. Finally, we find no obvious correlation between overdensity and the bars in our sample, showing that galactic bars are not obviously linked to the large-scale structure of the universe.

Subject headings: methods: statistical - galaxies: elliptical and lenticular, cD - galaxies: halos galaxies: spiral - galaxies: structure - large-scale structure of the universe
\end{abstract}

\section{INTRODUCTION}

The presence of bars in galaxies is a feature that was noticed from the earliest studies of external galaxies (Hubble 1936; de Vaucouleurs 1963). More recent estimates on the fraction of barred disk galaxies fluctuate between one- to two-thirds, depending on the method to identify bars and the inclusion or not of weak bars (Mulchaey \& Regan 1997; Knapen et al. 2000; Eskridge et al. 2000; Marinova \& Jogee 2007; Sheth et al. 2008; Cameron et al. 2010; Lee et al. 2012a; Oh et al. 2012). The large abundance of stellar bars in local galaxies has triggered interest on the involvement of these features on secular evolution (Sellwood \& Wilkinson 1993; Kormendy \& Kennicutt 2004; Cheung et al. 2013). Given that stellar bars break the radial symmetry of galaxies, they are particularly efficient in redistributing matter and angular momentum between different components, namely stars, gas and dark matter (Hohl 1971; Sellwood 1980; Tremaine \& Weinberg 1984; Weinberg 1985; Debattista \& Sellwood 2000; Athanassoula 2002, 2003; Martinez-Valpuesta et al. 2006). Clear examples of redistribution of material driven by bars is the fuel of gas inward (Shlosman et al. 1989; Friedli \& Benz 1993), resulting in accumulation of material that might be used as a build-up of disk-like bulges or pseudo-bulges (Kormendy \& Kennicutt 2004; Athanassoula 2005; Heller et al. 2007). Given the collisional nature of gas, this component can effectively lose energy during shocks and

\footnotetext{
sodi@kias.re.kr,leech@shao.ac.cn

${ }^{1}$ Partner Group of the Max Planck Institute for Astrophysics at the Shanghai Astronomical Observatory and Key Laboratory for Research in Galaxies and Cosmology of Chinese Academy of Sciences, Nandan Road 80, Shanghai 200030, China

2 School of Physics, Korea Institute for Advanced Study, Dongdaemun-gu, Seoul 130-722, Republic of Korea
}

flow inward (Shlosman et al. 1989; Friedli \& Benz 1993), which help to explain the high concentrations of molecular gas found on barred galaxies (Sakamoto et al. 1999; Sheth et al. 2005), as well as the younger stellar populations and higher star formation rates in the bulges of barred galaxies when compared with unbarred ones (e.g., Coelho \& Gadotti 2011; Wang et al. 2012). As a result, the overall structure and morphology of barred galaxies gets shaped by the effects of stellar bars.

At the same time, bar formation and evolution depends on many physical properties of hosting galaxies. In general, bars are more frequently found in massive, red galaxies with prominent bulges and overall early-type morphologies (Sheth et al. 2008; Weinzirl et al. 2009; Hoyle et al. 2011; Masters et al. 2011; Lee et al. 2012a; Cheung et al. 2013). Late-type, less massive, blue galaxies also show the presence of stellar bars, but their frequency is low and the bars they exhibit are shorter in size (Elmegreen \& Elmegreen 1985; Erwin 2005; Aguerri \& González-García 2009; Lee et al. 2012a). Masters et al. (2012) found that the bar fraction is significantly lower in gas-rich disk galaxies than in gas-poor ones, a result in agreement with theoretical expectations from Athanassoula et al. (2013), where they find in their simulations that bars form later in gas-rich systems, and after they form, they grow more slowly than in poor-gas systems, hence predicting that the bar likelihood should be higher in galaxies with low gas content. Using numerical simulations of spinning dark matter halos, Long et al. (2014) demonstrate that bar growth in strength and size, is strongly suppressed on systems with spin parameter $\lambda \gtrsim 0.03$, giving support to the observational finding by Cervantes-Sodi et al. (2013), where they reached this same conclusion estimating $\lambda$ for the same galaxy sample 
employed on the present study, and finding that strong bars are confined to reside in galaxies with low to intermediate values of $\lambda$, while weak bars are preferentially found in rapidly spinning systems. More recently, Cervantes Sodi et al. (2014) explored the dependence of the bar fraction on the stellar-to-halo mass ratio $\left(\mathrm{M}_{*} / \mathrm{M}_{\mathrm{h}}\right)$ for central disk galaxies finding, in agreement with theoretical studies (Ostriker \& Peebles 1973; Efstathiou et al. 1982; Christodoulou et al. 1995; DeBuhr et al. 2012), that bars are more common in galaxies with high $\mathrm{M}_{*} / \mathrm{M}_{\mathrm{h}}$ values, and exploring the bar fraction in the $\mathrm{M}_{\mathrm{h}} \mathrm{vs}_{*}$ plane, they find that the dependence is stronger considering a relation with the form $f_{\mathrm{bar}}=f_{\mathrm{bar}}\left(\mathrm{M}_{*}^{\alpha} / \mathrm{M}_{\mathrm{h}}\right)$ with $\alpha=1.5$.

Although secular evolution has proven to be an effective driver of evolution, it is by no means the only way in which a galaxy can transform. Regarding the formation and growth of bars, early simulations of interacting disk galaxies by Noguchi $(1987,1988)$ show that selfgravitating disks that were expected to be stable against the development of a bar, once perturbed by the tidal force of a companion, develop prominent spiral structures, that at the center resemble bar structures that are able to induce gas infall into the nucleus when gas dissipates energy via cloud-cloud collisions. Similar results are reported by Byrd \& Valtonen (1990) for the case of tidal forces exerted by a cluster potential on simulated disk galaxies. Although the configuration of the interaction changes the final properties of the bar, some groups found that in general, it enhances the formation of the bar (Gerin et al. 1990; Miwa \& Noguchi 1998), while others report that the opposite is also possible: the bar can loose angular momentum and mass and debilitate after the interaction (Sundin \& Sundelius 1991; Sundin et al. 1993). There seem to be also controversy about the different effects of prograde and retrograde interactions (Romano-Díaz et al. 2008; Aguerri \& González-García 2009).

Ultimately, the effect of environment on the formation and evolution of bars needs to be addressed through observational evidence. Thompson (1981) found a larger fraction of barred galaxies in the core of the Coma cluster and Giuricin et al. (1993) reported a higher recurrence of early-type barred spirals in high local density environments. Later studies of local (Andersen 1996; Eskridge et al. 2000) and intermediate-redshift clusters (Barazza et al. 2009) showed similar findings and, more recently, Méndez-Abreu et al. (2012) proved that by taking into account the different luminosity ranges, it is possible to detect different effects of the environment for luminous and faint galaxies located in clusters or in the field. They propose that interactions in bright disk galaxies that are stable enough against interactions trigger bar formation, while for faint galaxies, interactions are strong enough to heat the disk inhibiting bar formation.

The opposite result-no evidence for a dependence of bar frequency on environment, comparing field and cluster galaxies-is also widely reported (van den Bergh 2002; Marinova et al. 2009; Aguerri \& González-García 2009; Méndez-Abreu et al. 2010; Giordano et al. 2011; Marinova et al. 2012). When the samples include only S0 galaxies, most of the time, a secondary dependence on environment is reported, with a higher frequency of barred S0 galaxies in clusters (Barway et al. 2011) and an increase in the bar fraction toward the cluster core (Lans- bury et al. 2014).

By computing projected redshift-space two-point cross-correlation functions (2PCCF) for a sample of nearly 1,000 galaxies from the SDSS, Li et al. (2009, hereafter L09) studied the clustering properties of barred galaxies finding that the clustering of barred and unbarred galaxies of similar stellar mass is indistinguishable and no evidence that bar formation is promoted by mergers or interactions. More recently, Skibba et al. (2012), also using clustering statistics, reported a positive correlation for bulge-dominated and barred galaxies to be found in denser environments on scales of $150 \mathrm{kpc}$ to a few megaparsecs than their unbarred and disk-dominated counterparts and argued that barred galaxies are often central galaxies in low-mass dark matter halos or satellites in more massive ones.

Lee et al. (2012a) also investigated the dependence of the barred galaxy fraction on environment, finding that once mass and color are fixed, the bar fraction is independent of the background density, but an influence of the nearest neighbor appears when the separation to the nearest neighbor is less than 0.1 times the virial radius of the neighbor. As the distance decreases, the bar fraction drops, regardless of the morphology of the neighbor-a possible indication that strong tidal interactions destroy bars or prevent their growth. Similar results were presented by Méndez-Hernández et al. (2011) comparing reduced samples of isolated galaxies and galaxies in pairs, showing that the subsample of galaxies in pairs presented a bar fraction of only $20 \%$, while that for isolated systems was $43 \%$. Using a much larger galaxy sample, Casteels et al. (2013) reported a similar result using the Galaxy Zoo 2 sample, with a decrease for the likelihood of identifying a bar in pairs with separations $\leq 30 h^{-1} \mathrm{kpc}$.

In this paper, we study the environment of galaxies with bars in the local universe, extending the analysis of L09 by using the new sample provided by Lee et al. (2012a), which is 30 times larger than the sample used by L09. When compared to previous studies of the same topic, our approach differs in the following ways.

- Instead of computing the auto-correlation of the barred galaxy sample, we compute the crosscorrelation of our sample with respect to reference samples of the general galaxy population from scales of a few tens of kiloparsecs up to a few tens of megaparsecs. This takes advantage of the much larger sample size and volume of the reference samples, allowing us to substantially increase the signal-to-noise ratio in our results, as well as allowing us to study the scale dependence of the clustering of barred galaxies in detail.

- We wish to isolate the link between environment and the presence or absence of a bar in galaxies, so the environment measurements of the barred galaxies are always compared to those of control samples of unbarred galaxies that are closely matched in galaxy properties known to be correlated with environment. According to previous studies of the clustering of galaxies (e.g. Li et al. 2006a), we consider stellar mass, optical color and internal structure, quantified by the surface stellar mass density and the concentration of the stellar light distribution, when constructing the control samples. 
- In addition to computing the $2 \mathrm{PCCF}$, we use three other statistics to characterize the environment of our galaxies. These include (1) the backgroundsubtracted average neighbor counts $\left(N_{c}\right)$ around the barred and unbarred galaxies as a function of the projected distance to neighboring galaxies, (2) the overdensity $(\delta)$ of the local environment of our galaxies estimated on $\sim 3 \mathrm{Mpc}$ scale, and (3) the membership of our galaxies in the SDSS group systems identified by Yang et al. (2007). The different statistics have their pros and cons in quantifying galaxy environment, and provide measurements that are complementary to each other.

This paper is organized as follows. Section 2 gives a brief description of the volume-limited sample, as well as the control and reference samples used in this study. In Section 3, we describe the methods used to characterize the environment of the galaxies in our sample. In Section 4, we present our general results. Finally, in Section 5 we summarize our results and present our conclusions. Throughout this paper, we assume a cosmology with a density parameter of $\Omega=0.3$, cosmological constant of $\Omega_{\Lambda}=0.7$, and Hubble constant of $H_{0}=70 \mathrm{~km}$ $\mathrm{s}^{-1} \mathrm{Mpc}^{-1}$.

\section{DATA}

\subsection{The sample of barred galaxies}

The sample of galaxies with bar identification used in our study comes from a previous work by Lee et al. (2012a). Here we give a brief description of the sample selection and morphology classification, and the reader is referred to Lee et al. (2012a) for the full description of the sample and comparisons with previous classifications (de Vaucouleurs et al. 1991; Nair \& Abraham 2010) and Park \& Lee (2014) for the publicly available catalog. The sample is a volume-limited sample selected from the Korea Institute for Advanced Study Value-Added Galaxy Catalog, which was constructed from the SDSS DR7 (Abazajian et al. 2009) by Choi et al. (2010) and consists of 33,391 galaxies with $r$-band absolute magnitudes brighter than $M_{r}=-19.5+5 \log h$ and spectroscopically measured redshift in the range $0.02 \leq z \leq 0.05489$. The segregation into early- and late-type galaxies is done by adopting the prescription by Park \& Choi (2005), where galaxies are divided according to their morphology in color versus color gradient and concentration index space plus an additional visual inspection. The identification of bars is done by visual inspection of $g+r+i$ combined color images from SDSS. Early-type galaxies are classified as either barred or unbarred galaxies. For the case of late-type galaxies, given that the classification is more robust for face-on systems, we limit our sample to galaxies with a minor-to-major axis ratio of $b / a>0.6$. For late-type galaxies presenting a bar, the bar is further classified as strong or weak according to its size-if the bar is larger than one quarter the size of the hosting galaxy, it is classified as a strong bar, otherwise it becomes a weak bar. Given that the bar fraction estimated by visual inspection of optical images is statistically the same as that estimated using automated methods such as ellipse fitting (Menéndez-Delmestre et al. 2007; Sheth et al. 2008) and that the computation of the bar fraction gives the same result using near-infrared images as it does when using optical (Whyte et al. 2002; MenéndezDelmestre et al. 2007; Sheth et al. 2008), we are confident that our results are reproducible even if the bar detection is obtained by using a different method.

We extract the physical quantities required for our work from two additional catalogs: the New York University Value Added Galactic Catalog (NYU-VAGC; Blanton et al. 2005) ${ }^{3}$ and the MPA/JHU SDSS database (Kauffmann et al. 2003; Brinchmann et al. 2004) ${ }^{4}$, both of which are based on SDSS DR7 and publicly available. These include stellar mass $M_{*}$, stellar surface mass density $\mu_{*}$, color $(g-r)$ and concentration index $C$. The stellar mass of a galaxy was estimated based on its redshift and the five-band SDSS photometry following the methodology detailed in Kauffmann et al. (2003) ${ }^{5}$. The surface stellar mass density of a galaxy is defined by $\mu_{*}=0.5 M_{*} / \pi R_{50, z}^{2}$, where $R_{50, z}$ is the radius enclosing $50 \%$ of the $z$-band flux, and the concentration index $C=R_{90, r} / R_{50, r}$, where $R_{90, r}$ and $R_{50, r}$ are the radii enclosing $90 \%$ and $50 \%$ of the total light in the $r$-band image of the galaxy. Our final sample includes 17,839 unbarred and 3,749 barred galaxies. In order to distinguish this sample from subsequent control samples, we will refer to it as $\mathrm{C} 0$ sample, which we will divide into eight different subsamples: $\mathrm{AU}(\mathrm{AB})$ includes all unbarred (barred) galaxies, EU (EB) includes only unbarred (barred) early-type galaxies, LU (LB) includes only unbarred (barred) late-type galaxies, SB includes only strongly barred late-type galaxies and WB includes weakly barred late-type galaxies.

\subsection{Control samples of unbarred galaxies}

Previous studies have shown that barred and unbarred galaxies present different stellar mass, color, and surface mass density distributions, and at the same time the clustering of galaxies strongly depends on all these properties (e.g. Li et al. 2006a).In order to isolate the effect of environment on bars, we construct a series of control samples of unbarred galaxies, and in what follows we compare our results of each barred-galaxy subsample always with those of the corresponding control sample. We compile two different control samples: $\mathrm{C} 2$ encompasses galaxies with matched stellar mass and color between barred and unbarred galaxies and C3 matches an extra quantity-the stellar surface mass density. The matching tolerances are $\triangle \log M_{*} \leq 0.08, \triangle(g-r) \leq 0.025, \triangle \log \mu_{*} \leq 0.08$.

\subsection{Reference galaxy samples}

We have constructed two reference samples from NYUVAGC: a spectroscopic reference sample, which is used to compute the projected two-point cross-correlation function $(2 \mathrm{PCCF}) w_{p}\left(r_{p}\right)$, and a photometric reference sample, which is used to calculate counts of close neighbors around our barred and unbarred galaxies. The spectroscopic reference sample is constructed from version dr72

\footnotetext{
3 http://sdss.physics.nyu.edu/vagc/

${ }^{4}$ http://www.mpa-garching.mpg.de/SDSS/

5 Note that the masses included in the current MPA/JHU database are based on fits to photometry and so are not identical to those of Kauffmann et al. (2003) where the masses are obtained from spectroscopic indices. See http://www.mpagarching.mpg.de/SDSS/DR7/mass_comp.html for detailed comparisons between the two masses.
} 


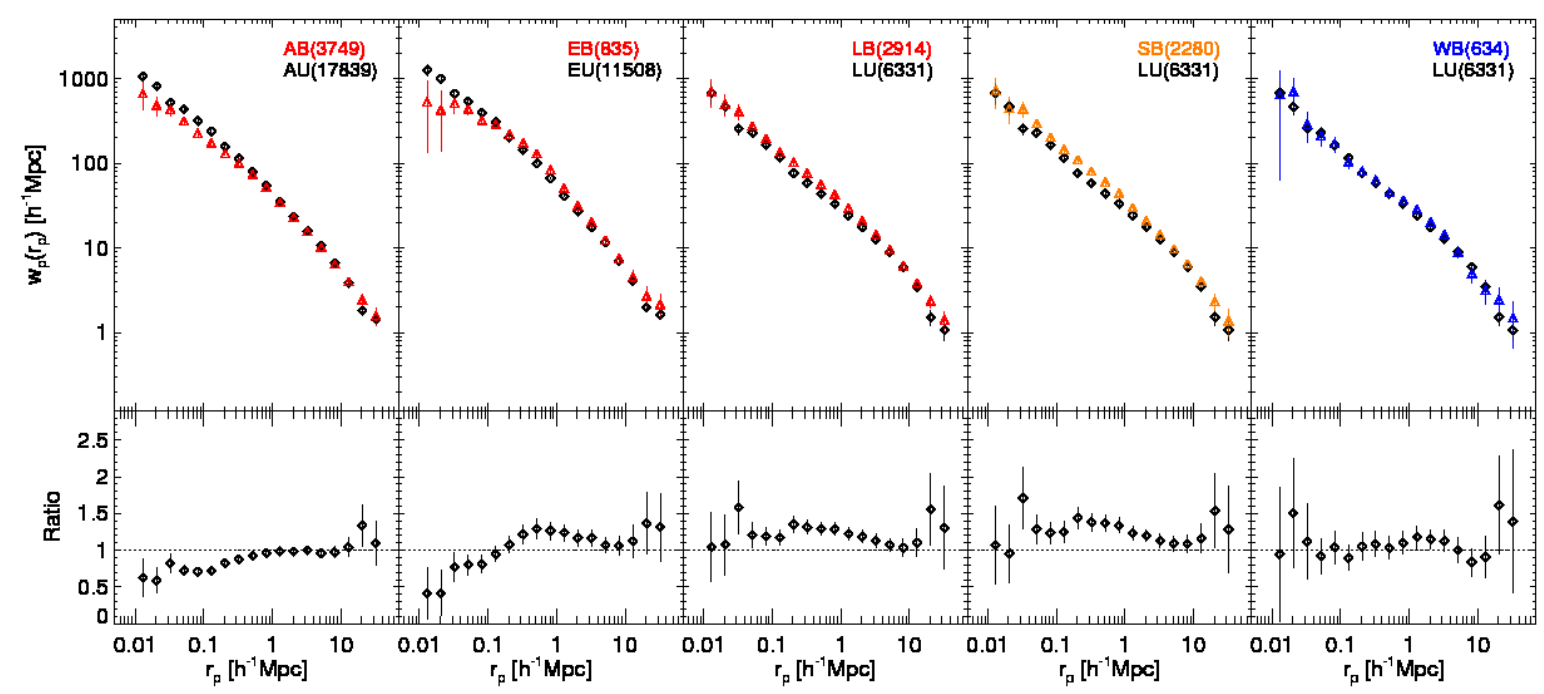

FIG. 1. - Projected 2PCCF for barred $w_{p}^{\text {bar }}\left(r_{p}\right)$ and unbarred $w_{p}^{\text {unbar }}\left(r_{p}\right)$ galaxies (top panels) and $w_{p}^{\text {bar }}\left(r_{p}\right)$ to $w_{p}^{\text {unbar }}\left(r_{p}\right)$ ratio (bottom panels) for the different subsamples of our parent galaxy sample C0. Panels from left to right correspond to the whole galaxy sample of barred (AB orange triangles) plus unbarred (AU black diamonds) galaxies, early-types barred (EB orange triangles) and unbarred (EU black diamonds), late-types barred (LB orange triangles) and unbarred (LU black diamonds), strongly barred late-types (SB yellow triangles) and LU, and weakly barred late-types (WB blue triangles) and LU.

of the NYU-VAGC, the current version built on the SDSS DR7 data, by selecting all galaxies with $r$-band apparent magnitude corrected for foreground extinction $r<17.6$ within the restricted redshift range of $0.01 \leq z \leq 0.2$ and the absolute magnitude range $-23 \leq M_{0.1_{r}} \leq-17$. Here $M_{0.1_{r}}$ is the $r$-band absolute magnitude corrected to its value at $z=0.1$. With these selection criteria, we are left with about half a million galaxies in our spectroscopic reference sample. The photometric reference sample is also constructed from NYU-VAGC version $\mathrm{dr} 72$ by selecting galaxies with $r$-band apparent magnitude down to $r<21$. The sample includes about 2.5 million galaxies.

\section{METHODOLOGY}

In this section we briefly describe the methods we will use to characterize the environment of the galaxies in our samples. For a full description of these methods, we refer the reader to the source papers where they are presented in detail (Li et al. 2006a,b; Yang et al. 2007; Jasche et al. 2010).

\subsection{Projected two-point cross-correlation function}

We use the projected redshift-space $2 \mathrm{PCCF}, w_{p}\left(r_{p}\right)$, to quantify the clustering properties of our galaxies. For this we have constructed a random sample that has the same selection effects as the spectroscopic reference sample following the method described in Li et al. (2006a). We cross-correlate each of our samples of barred galaxies, as well as the corresponding control sample of unbarred galaxies, with respect to both the spectroscopic reference sample and the random sample, and then define $w_{p}\left(r_{p}\right)$ as a function of the projected separation $r_{p}$ by the ratio of the two pair counts minus one. A careful correction for the effect of fiber collisions is implemented by comparing the angular $2 \mathrm{PCF}$ of the spectroscopic sample with that of the parent photometric sample (see Li et al. 2006b, for details). Errors of the $w_{p}\left(r_{p}\right)$ measurements are estimated using the bootstrap resampling technique (Barrow et al. 1984), for which a total of 100 bootstrap samples are generated based on the real sample. Details about our methodology for computing the correlation functions and for constructing the random sample can be found in Li et al. (2006a).

\subsection{Close neighbor counts}

We count the number of galaxies in the photometric reference sample in the vicinity of the galaxies in our samples, and we make a statistical correction for the effect of chance projections by subtracting the average count around randomly placed galaxies. When compared to the $2 \mathrm{PCCF}$, close neighbor counts are not affected by incompleteness on small scales caused by the fiber collisions. In addition, since the photometric sample is much deeper than the spectroscopic sample, by computing background-subtracted neighbor counts in the photometric sample one is able to include much fainter companion galaxies, thus probing the effect of close companions over a broader range of mass ratios.

\subsection{Group catalog}

To account for the environment of galaxies in groups, we make use of the group catalog of Yang et al. (2007), constructed from the sample dr72 of the NYU-VAGC. The groups of galaxies are identified using a modified version of the halo-based group-finding algorithm developed in Yang et al. (2005) and applied to a sample with redshifts in the range $0.01 \leq z \leq 0.20$ and with a redshift completeness greater than 0.7. From this extensive catalog, we use only galaxies identified in groups with at least three members, from which we are left with a final sample of nearly 2,000 galaxies. For each group system, we take the most massive galaxy as the central galaxy, and the rest as satellites.

\subsection{Large-scale overdensity}






FIG. 2.- Same as the Figure 1, but using control sample C2 where the galaxies share a common distribution of stellar mass $M_{*}$ and color $\mathrm{g}-\mathrm{r}$.

To account for the environment at large scales, we make use of a non-linear, non-Gaussian, full Bayesian large-scale structure analysis of the cosmic density field based on the SDSS DR7 by Jasche et al. (2010), where the authors use a Bayesian sampling algorithm in order to obtain the extremely high dimensional non-Gaussian, non-linear log-normal Poissonian posterior of the threedimensional density field. The reconstruction of the field is made over a $750 \mathrm{Mpc}$ cube, taking into account the angular and radial selection functions of the SDSS, and a proper treatment of a Poissonian shot noise contribution for an effective resolution of the order of $\sim 3 \mathrm{Mpc}$.

\section{RESULTS}

\subsection{Cross-correlation functions}

In Figure 1, we show the $2 \mathrm{PCCF} w_{p}\left(r_{p}\right)$ measured for our main galaxy sample $\mathrm{C} 0$. In each panel we present $w_{p}(r)$ for barred $w_{p}(r)^{b a r}$ and unbarred $w_{p}(r)^{\text {unbar }}$ galaxies for the different subsamples in consideration (from left to right: all the galaxies in the sample, early-types, late-types, strongly barred late-types and weakly barred late-types). For clarity we also include the amplitude ratio $w_{p}(r)^{b a r}$ to $w_{p}(r)^{\text {unbar }}$ for each case.

At first sight, it is clear that the clustering of our barred and unbarred samples are very similar for all the subsamples, although some differences appear when looking carefully at each case. Starting with the extreme left panel, which includes all the galaxies in our sample, we notice that unbarred galaxies are more strongly clustered than barred ones on scales below $\sim 1 \mathrm{Mpc}$. If we include only early-types in our analysis (second panel), the clustering ratio between barred and unbarred systems changes, presenting a pick at around $1 \mathrm{Mpc}$ and positive values at larger scales. Only on scales bellow $100 \mathrm{kpc}$ does the ratio becomes less than unity. When we turn to the case of late-type galaxies (middle panel), barred galaxies appear to be more strongly clustered than their unbarred counterparts at almost all the scales probed. If we distinguish between strong (fourth panel) and weak (fifth panel) bars, we see that the signal seen for the case of late-type galaxies mostly comes from the clustering of strongly barred galaxies, which is natural given that they are more numerous in our sample than weakly barred galaxies.

At this point, Figure 1 seems to indicate that the clustering of barred and unbarred galaxies is different, but we need to be careful to drive conclusions at this stage given the following two facts. First, previous studies of galaxy clustering have well established that clustering strongly depends on galaxy properties including mass, color and morphological type (e.g. Li et al. 2006a; Zehavi et al. 2011). Second, it is also well known that the presence or absence of bars is not the only morphological difference between our barred and unbarred samples. For instance, previous works (Barazza et al. 2008; Nair \& Abraham 2010; Lee et al. 2012a) show that the fraction of barred galaxies is higher in massive red galaxies than in less-massive blue systems. Massive red galaxies are more strongly clustered than less-massive, blue galaxies. To normalize out this effect we make use of our control samples C2 and C3. As described in Section 2.1, for the case of $\mathrm{C} 2$ we have restricted our samples so that each subsample of barred galaxies has the same distribution in stellar mass and color as the corresponding subsample of unbarred galaxies, while for the case C3 the subsamples of barred and unbarred galaxies being compared are additionally required to have the same distribution in stellar surface mass density.

Measurements of the 2PCCF for our $\mathrm{C} 2$ and $\mathrm{C} 3$ samples are shown in Figures 2 and 3, respectively. For latetype galaxies, the different clustering behaviors between barred and unbarred galaxies seen in Figure 1 disappear when the samples being compared are closely matched in stellar mass, color and surface mass density, with the ratio $w_{p}\left(r_{p}\right)^{\text {bar }}$ to $w_{p}\left(r_{p}\right)^{\text {unbar }}$ consistent with unity within error bars on all scales probed. The same result holds if we further split our sample of barred late-type galaxies into strongly barred and weakly barred galaxies according to the size of their bars. This is consistent with the expectation that the relatively strong clustering of 


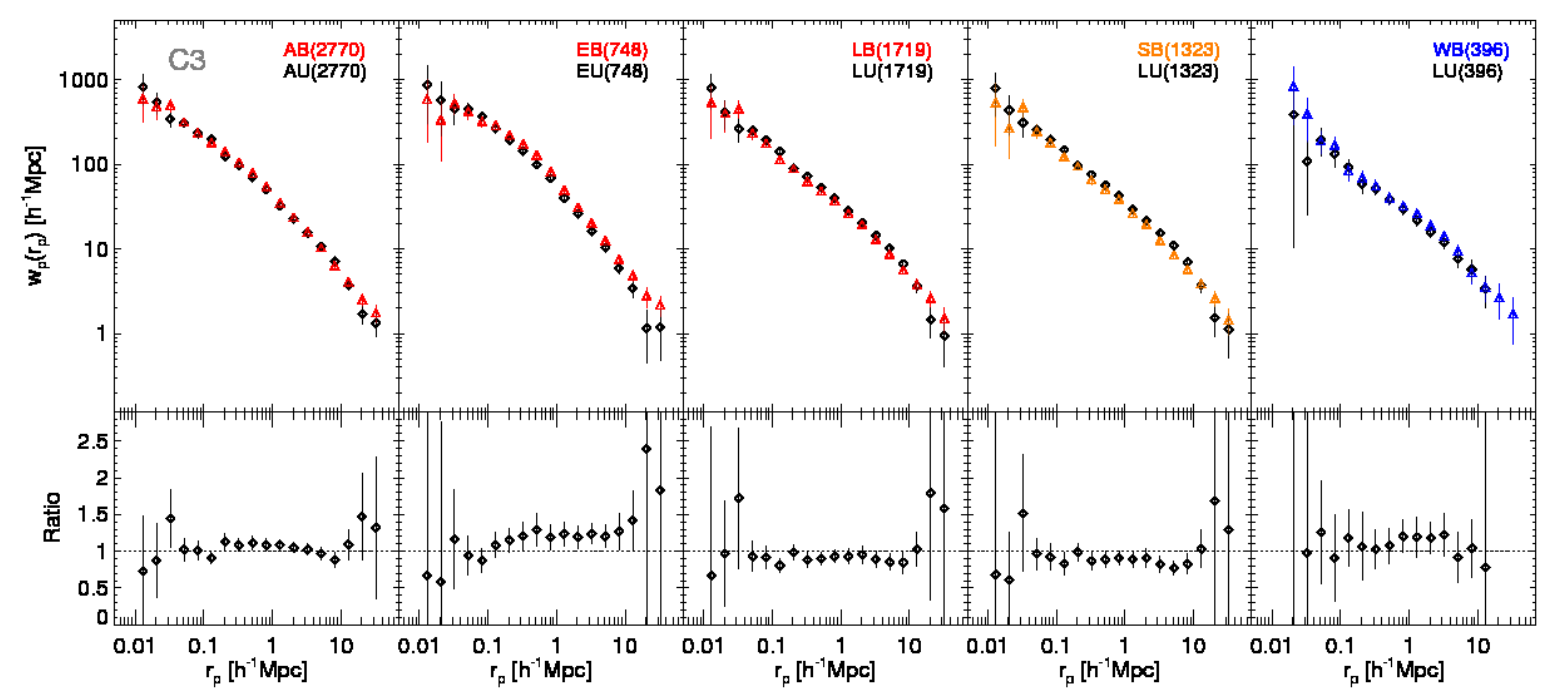

Fig. 3. - Same as the Figure 1, but using control sample C3 where the galaxies share a common distribution of stellar mass $M_{*}$, color $\mathrm{g}-\mathrm{r}$, and stellar surface mass density $\mu_{*}$.



FIG. 4. - Histograms for stellar mass $M_{*}, g-r$ color, stellar surface mass density $\mu_{*}$ and the concentration index C for control samples C0 (dotted lines) and C3 (solid lines). The first line corresponds to the whole sample, the second line to early-types, and the third line to late-types.

late-type, barred galaxies seen for the full sample (i.e. sample $\mathrm{C} 0$ ) is indeed attributed to the fact that the subset of barred galaxies with late-type morphology in $\mathrm{C} 0$ are more massive and redder, thus more strongly clustered, when compared to the unbarred late-type galaxies. This result implies that the presence of a bar in late-type galaxies is not obviously linked with their clustering properties as quantified by the $2 \mathrm{PCCF}$, confirming the earlier finding of Li et al. (2009) that was based on a much smaller sample.

For early-type galaxies, in contrast, the difference in the 2PCCF between barred and unbarred galaxies is still significantly seen for scales larger than a few hundred kiloparsecs, even when the samples are closely matched in mass, color, and surface mass density. Considering that the clustering of galaxies depends not exclusively on these parameters, we compare the distributions of stellar mass, $\mathrm{g}-\mathrm{r}$ color, surface mass density, and concentration index $C$ for samples $\mathrm{C} 0$ and $\mathrm{C} 3$ in Figure 4 . In the case of $\mathrm{C} 3$, the distributions for $M_{*}, \mathrm{~g}-\mathrm{r}$ and $\mu_{*}$ are indistinguishable between barred and unbarred galaxies, regardless of their morphological type, but the concentration 


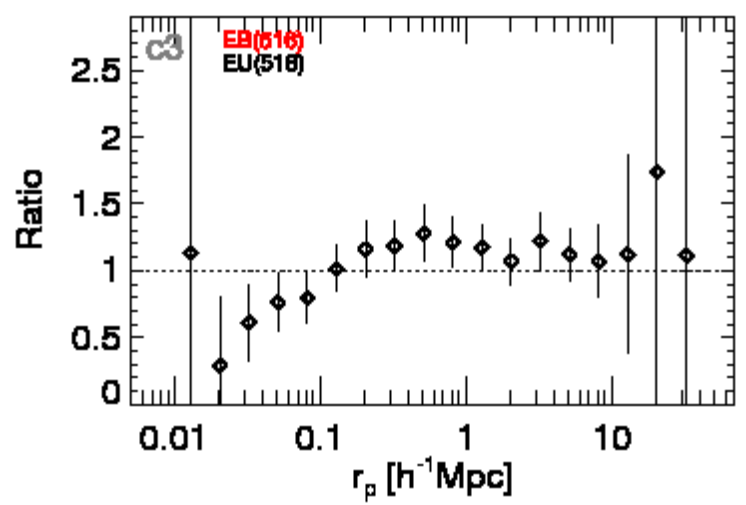

FIG. 5.- Same as the Figure 1, but using control sample C3 for early-type galaxies with $2.8 \leq C \leq 3.5$.

index $C$ follows different statistical distributions between barred and unbarred systems, especially for the case or early-type galaxies (see the rightmost panels). In order to further isolate the link between bars and the clustering of early-type galaxies, we remove the dependence of clustering on concentration index from our analysis by restricting ourselves to a narrow range of concentration index $2.8 \leq C \leq 3.5$, and we repeat the same analysis for early-type galaxies as we did above for the case of C3. The result is presented in Figure 5. Although the difference on large scales ( $>1 \mathrm{Mpc}$ ) becomes less significant, the stronger clustering of barred galaxies still persists on intermediate scales, at around $500 \mathrm{kpc}$. As shown in $\mathrm{Li}$ et al. $(2008 \mathrm{a}, \mathrm{b})$, on these scales the $2 \mathrm{PCCF}$ is dominated by pairs of galaxies hosted by the same dark matter halo. Thus the difference in 2PCCF between barred early-type galaxies and unbarred early-type galaxies implies that the two types of galaxies are distributed in their host dark matter halo in different ways. We will come back to this point in Section 4.4.

\subsection{Close neighbor counts}

A problem with computing the $2 \mathrm{PCCF}$ on very small scales using our spectroscopic sample is the effect of fiber collisions. Although we implemented a statistical correction that is proved correct on average, it might still introduce systematic effects in our study. In this subsection we investigate the clustering of our samples by computing the background-subtracted neighbor counts, $N_{c}\left(r_{p}<R_{p}\right)$, which is by definition the number of galaxies in the photometric reference sample within the projected radius $R_{p}$ of the barred or unbarred galaxies, with the effect of chance projection being statistically corrected (see Section 2). When compared to the $2 \mathrm{PCCF}$ analyzed above, this quantity doesn't suffer from the fiber collision effect on small scales. It also allows us to explore the effect of fainter companion galaxies. For this analysis we only consider our C3 samples where the dependence of clustering on mass, color and internal structure of galaxies has been taken into account.

In Figures 6 and 7 , we plot $N_{C}$, measured within a given value of projected radius $R_{P}$, for barred and unbarred galaxies in sample C3. As in the previous subsection, we make comparisons for barred and unbarred galaxies for given morphological type with their corresponding ratios of $N_{C}$ for barred galaxies relative to those of unbarred ones. Figure 6, top to bottom, shows the results for the whole sample, the early-type, and latetype subsamples, respectively, and in Figure 7, we split present again the result for late-types as well as the result from splitting the sample into strong and weak bars. Panels in both figures, from left to right, show the results for different apparent $r$-band limiting magnitude applied to the photometric reference sample ranging from $r_{\text {lim }}=18$ for the leftmost panels to $r_{\text {lim }}=21$ for the rightmost panels.

In Figure 6 (top panels), where we compare the neighbor counts around all the barred galaxies and the control sample of unbarred galaxies, we see that barred galaxies have less companions on scales of $\lesssim 50 \mathrm{kpc}$ than unbarred galaxies, while on scales of $\gtrsim 100 \mathrm{kpc}$, barred galaxies present more neighbors than their unbarred counterparts. When we split the galaxies according to their morphological type, we find that the differences on the small and large scales, found in the top panels for the barred galaxies as a whole, are essentially contributed by late-type and early-type galaxies separately. As the second row of panels show, the higher neighbor count on large scales is an effect seen only for early-type galaxies, indicating that barred, early-type galaxies are located in higher density regions than unbarred, early-type galaxies of similar mass, color and internal structure. This confirms what we find above from the 2PCCF measurements, where the same sample of barred galaxies shows higher clustering amplitude on intermediate scales than the control sample of unbarred galaxies. When the comparison is limited to late-type galaxies, the difference on large scales is no longer significant, while at the smallest scales we see clearly the drop in $N_{c}$, suggesting that the same effect found for the full sample is driven by late-type galaxies.

Figure 7 presents the result for late-type galaxies only, making the distinction between strong and weak bars. The large number of strongly barred galaxies in our sample helps to improve the statistics and reduce the statistical errors in our measurements, but even with large error bars, if we look at the result for weakly barred galaxies we see that the drop of average neighbor counts is noticeable at even larger scales, up to a few hundred kiloparsecs. A possible explanation for this effect at larger scales for the case of weakly barred galaxies might be related with these systems being less massive than those galaxies hosting strong bars (Lee et al. 2012b; Cervantes-Sodi et al. 2013), which would make them less stable against interactions with massive neighbors even at large separations.

Finally, we notice that in all the cases we have considered, the differences in the neighbor count change very little as one goes to fainter and fainter limiting magnitudes. This implies that the excess or drop in neighbor counts seen above is contributed primarily by relatively bright companions with $r<18$, but not the fainter companions.

\subsection{Galaxy group catalog}

Our results studying the $2 \mathrm{PCCF}$ and average number counts for our sample of galaxies indicate that barred early-type galaxies present a higher clustering amplitude 


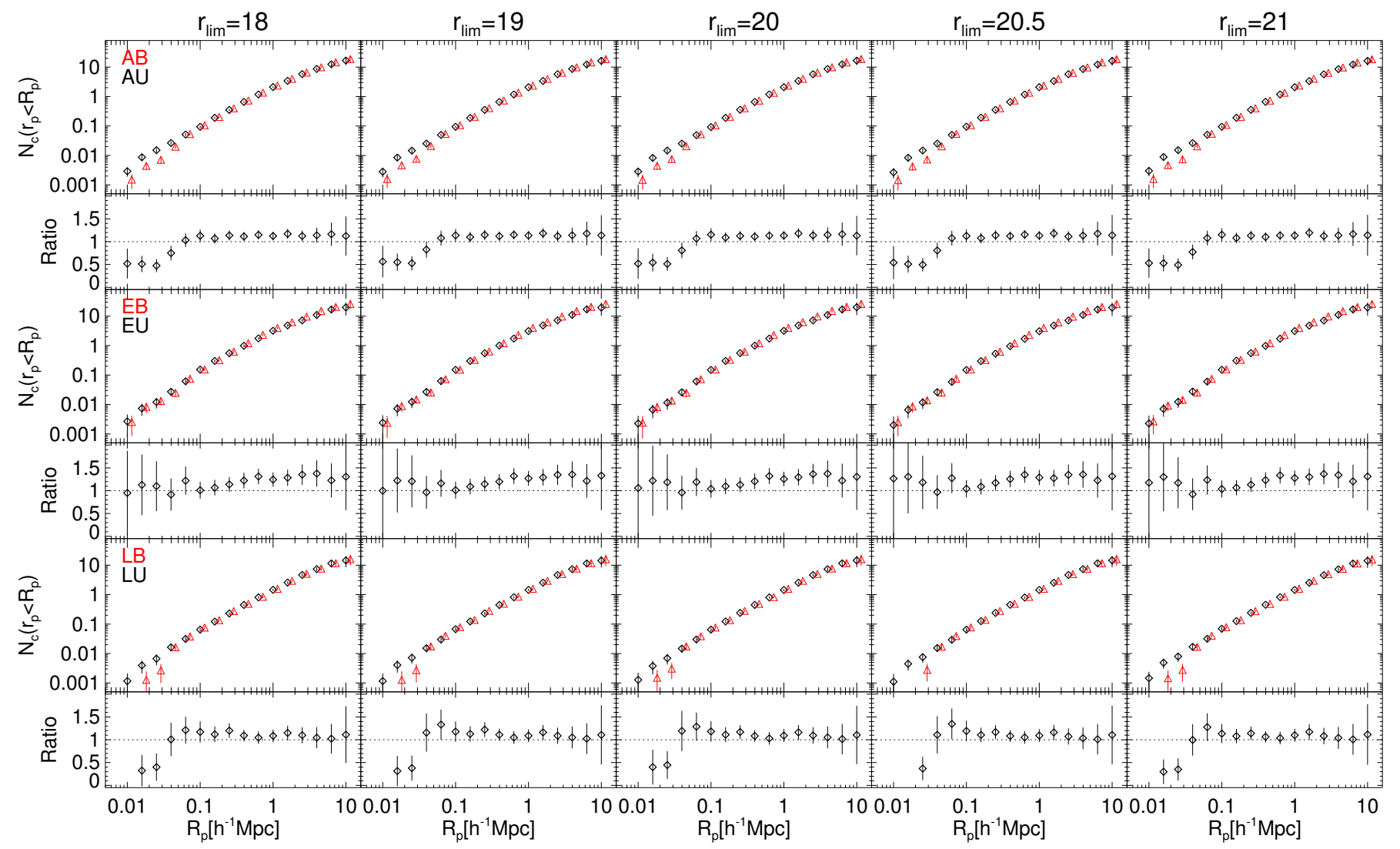

FIG. 6.- Average counts of galaxies in the photometric sample within a given projected radius $\mathrm{R}_{P}$ from the galaxies in the C3 subsamples. Each line corresponds to different apparent magnitude limits in the $r$ band $(r \leq 18,19,20,20.5,21)$ for the galaxies in the photometric sample.

and a larger number of companion galaxies on scales from a few hundred kiloparsecs to several megaparsecs when compared with unbarred ones. In particular, Figure 5 reveals a maximum at $\sim 500 \mathrm{kpc}$ in the ratio of the $2 \mathrm{PCCF}$ between barred and unbarred early-type galaxies, with the ratio decreasing at both smaller and larger scales. This implies that the 2PCCFs of the two samples are different not only in amplitude, but also in shape on these scales. As shown in Li et al. (2006b), the amplitude of $2 \mathrm{PCCF}$ on scales larger than a few megaparsecs is a direct measure of the mass of host dark matter halos, while the shape of the $2 \mathrm{PCCF}$ on a few $\times 100 \mathrm{kpc}$ is sensitive to the relative fraction of central and satellite galaxies in the galaxy sample. Our result here thus suggests that early-type barred galaxies are expected to be found more frequently in satellite galaxies than in central galaxies.

To further explore this possibility we make use of the group catalog of Yang et al. (2007) in order to identify central and satellite galaxies in our sample. As described in the previous section, after matching our main sample with the group catalog and only keeping galaxies in groups with three or more members, our final sample reduces to nearly 2,000 galaxies. From this reduced sample we find that the ratio of central-to-satellite systems for barred and unbarred late-type galaxies is very similar, about 27\% (see also Cervantes Sodi et al. (2014)). For early-type galaxies this ratio is significantly different: $24 \%$ for unbarred and only $16 \%$ for barred galaxies. Although the small sample size doesn't allow us to do a more detailed analysis, the different central-to-satellite galaxy number ratio for early-type galaxies is already telling us that early-type barred galaxies in our sample are indeed more commonly found as satellites in galaxy groups, reinforcing our hypothesis from the results using 2PCCFs and neighbor counts.

\subsection{Large-scale overdensity}

Finally, we have also examined the overdensity of the large-scale environment of our barred and unbarred galaxies. For this, we use the overdensity $\delta$ estimated over a scale of $\sim 3 \mathrm{Mpc}$ by Jasche et al. (2010) through a reconstruction of the three-dimensional density field in the local universe based on the SDSS DR7 spectroscopic galaxy sample, as described on Section 3.4. The results are shown in Figure 8, where we compare the cumulative distributions of the different subsamples of our control sample $\mathrm{C} 3$ as a function of $\ln (2+\delta)$. As can be seen, the distributions of barred and unbarred galaxies are almost identical in all cases. We conclude that the presence of a bar in galaxies is unlikely correlated with the overdensity of local environment on scales of $\sim 3 \mathrm{Mpc}$. This is in good agreement with what we find above from the 2PCCFs and close neighbor counts on large scales.

\section{SUMMARY AND DISCUSSION}

We have presented an extensive study of the environment of galaxies with bars in the low-redshift universe, which makes use of a volume-limited sample of $\sim 30,000$ galaxies from the SDSS with visually determined morphological classifications and bar identifications. We use 


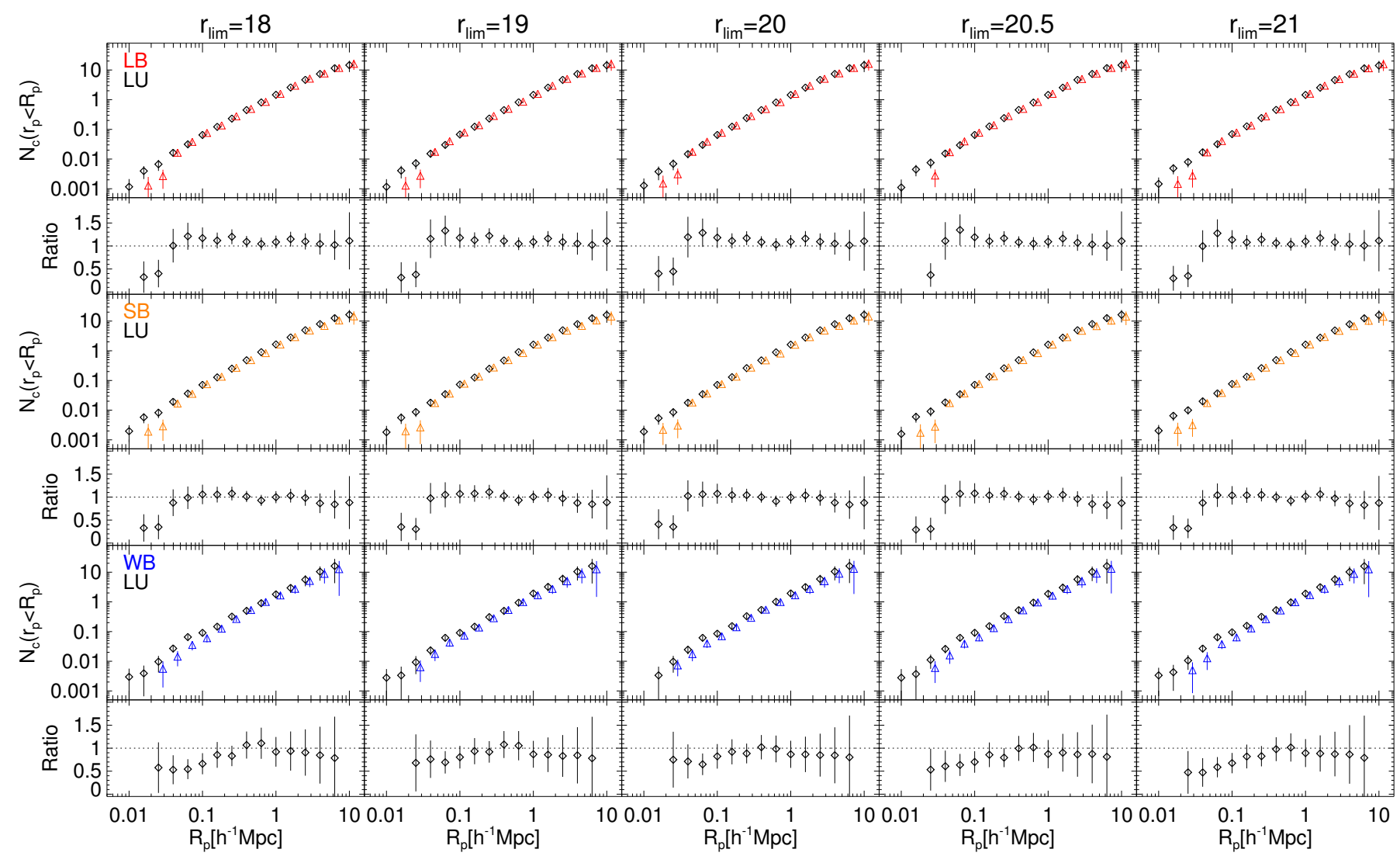

FIG. 7. - Ratio of average neighbor counts of barred to unbarred galaxies for C3 within a given apparent magnitude limit in the $r$ band $(r \leq 18,19,20,20.5,21)$.

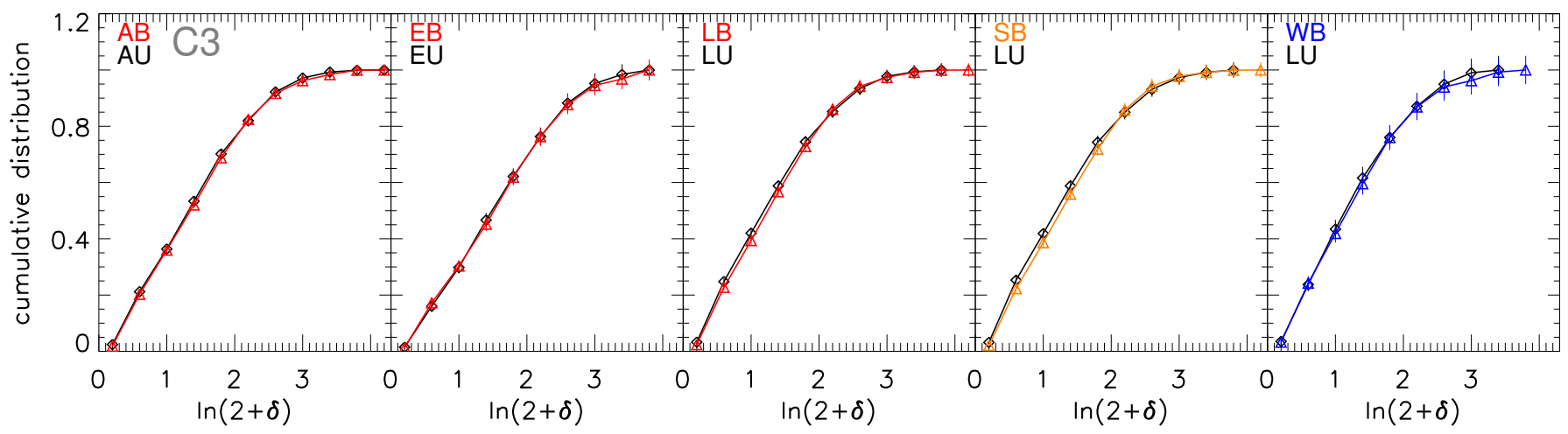

FIG. 8.- Cumulative distribution of barred (color line) and unbarred (black line) galaxies as a function of the overdensity parameter $\delta$ for the different subsamples of C3.

four distinct statistics to characterize the environment of the barred galaxies in our sample: the $2 \mathrm{PCCF} w_{p}\left(r_{p}\right)$ of our galaxies with respect to a spectroscopic reference sample, the background-subtracted average counts $N_{c}\left(R_{p}\right)$ of neighboring galaxies in a photometric reference sample, the membership of our galaxies in the SDSS group systems, and the overdensity $\delta$ of the local environment at $\sim 3 \mathrm{Mpc}$ scale. We segregate our galaxies into early- and late-types to study if differences arise between the two different morphological types. For the late-type subsample, we further distinguish two types of barred galaxies, those with strong and weak bars, according to their size. We apply the four statistics to the subsam- ples of barred galaxies, and compare the results to the same statistics obtained for control samples of unbarred galaxies that are closely matched to the barred galaxy samples in stellar mass, color, and internal structure in order to normalize out the dependence of environment on these parameters.

The first thing we notice measuring $w_{p}\left(r_{p}\right)$, once we remove any dependence on stellar mass, color and stellar surface mass density, is that the clustering for barred and unbarred galaxies are very similar, with only minor differences. The differences arise when we split the galaxies in our sample according to their morphological types. For the case of late-type galaxies, the 2PCCF does not 
depend on the presence or absence of a bar, confirming the previous result by Li et al. (2009). The barred earlytype subsample shows a higher amplitude for $w_{p}(r)$ on scales from a few hundred kiloparsecs to $1 \mathrm{Mpc}$, when compared with unbarred galaxies, very similar to the result reported by Skibba et al. (2012), who found a higher likelihood for barred and bulge-dominated galaxies on denser environments on scales of $150 \mathrm{kpc}$ to a few megaparsecs, than unbarred, disk-dominated ones. At these intermediate scales, the correlation function is dominated by the one-halo term, which would indicate that barred early-type galaxies are more frequently found as satellite systems. To explore this idea further, we made use of the Yang et al. (2007) group catalog to identify our galaxies in groups as centrals or satellites, finding that the percentage of barred early-type galaxies was significantly higher for satellites compare with the percentage for centrals. In contrast, the percentage of barred latetype galaxies is the same for central as for satellite galaxies, in agreement with recent results by Cervantes Sodi et al. (2014).

With our barred early-type sample composed by S0 galaxies, this result is also in agreement with Barway et al. (2011) who found that S0 galaxies in clusters present a higher bar fraction than their counterparts in the field, and Lansbury et al. (2014), who analyzed a reduced sample of S0 galaxies in Coma and found an increase in the bar fraction from the outskirts of the cluster toward the core. A possible framework to explain this trend is that these S0 galaxies were originally spirals that were transformed by ram pressure stripping with the intracluster medium, loosing their cold interstellar gas to the environment, causing a fading of the disk and subsequently a morphology change, but preserving their stellar features such as the bar. This mechanism is expected to work only for faint lenticular galaxies (Boselli \& Gavazzi 2006; Barway et al. 2009), which are not able to retain their gas, while bright S0s presumably form through other processes (e.g., mergers). Interestingly, our barred lenticulars are mostly faint galaxies, with $\mathrm{M}_{r}>-21.5$. With a higher bar fraction for spirals than for lenticulars (Laurikainen et al. 2009), this could explain the higher amplitude found for the correlation function in our sample of barred early-type galaxies on scales that correspond to satellite systems of more massive halos.

Given that our estimate for the $2 \mathrm{PCCF}$ suffers the effect of fiber collisions at small scales, we turned to close neighbor counts to study the clustering of barred galaxies at small scales. In this case, it is the barred late-type subsample that shows a systematic trend of having an average lower number of neighbors on scales of $\leq 50 \mathrm{kpc}$ when compared with the control sample of unbarred late-type galaxies. The effect seems more dramatic for the case of weakly barred galaxies, where the deficiency appears at larger scales close to $100 \mathrm{kpc}$. This finding may indicate that tidal interactions destroy and/or disfavor bar growth instead of enhancing it as expected from results of numerical simulations. As weak bars are more commonly found in fainter, less-massive galaxies than strong bars (Lee et al. 2012b; Cervantes-Sodi et al. 2013), the fact that we find the effect of nearby companions at larger scales for the case of weak bars may be not unexpected: due to their lower mass their gravitational potential is also weaker and they are more fragile to disturbances than their more massive counterparts. In addition, the decline of the average close neighbor count around the barred galaxies in our sample is found to be weakly dependent on the limiting magnitude of the photometric sample, implying that the effect is primarily driven by relatively bright companion galaxies, with little contribution from companions fainter than $r=18$.

Our result echoes previous studies that report a decrease on the bar fraction for disk galaxies when the separation to the nearest neighbor becomes smaller than 0.1 times the virial radius of the neighbor (Lee et al. 2012a), a lower fraction of barred galaxies in close pairs when compared with isolated galaxies (MéndezHernández et al. 2011) and a decrease on the likelihood of identifying a bar in pairs with small separations (Casteels et al. 2013). In any case, we only find indications of suppressed formation/growth of stellar bars in galaxies that might be undergoing some kind of interaction with a close neighbor, with no evidence of environmental stimulation, in opposition with earlier studies (Elmegreen et al. 1990).

Finally, we do not find any dependence of the likelihood of galaxies hosting bars on the large-scale environment, as accounted by the overdensity parameter $\delta$, estimated through a reconstruction of the three-dimensional cosmic web posterior (Jasche et al. 2010). This result suggests that galactic bars are not obviously linked to the largescale structure of the universe.

The authors thank the anonymous referee for comments that improved the paper. We are grateful to Changbom Park for providing the galaxy sample used in this study and Ramin Skibba, Edmond Cheung, and Jairo Mendez-Abreu for helpful discussions. This work is sponsored by NSFC (grant Nos. 11173045, 11233005, 11325314, 11320101002) and the Strategic Priority Research Program "The Emergence of Cosmological Structures" of CAS (grant No. XDB09000000). Funding for the SDSS and SDSS-II has been provided by the Alfred P. Sloan Foundation, the Participating Institutions, the National Science Foundation, the U.S. Department of Energy, the National Aeronautics and Space Administration, the Japanese Monbukagakusho, the Max Planck Society, and the Higher Education Funding Council for England. The SDSS Web site is http://www.sdss.org/. The SDSS is managed by the Astrophysical Research Consortium for the Participating Institutions. The Participating Institutions are the American Museum of Natural History, Astrophysical Institute Potsdam, University of Basel, University of Cambridge, Case Western Reserve University, University of Chicago, Drexel University, Fermilab, the Institute for Advanced Study, the Japan Participation Group, Johns Hopkins University, the Joint Institute for Nuclear Astrophysics, the Kavli Institute for Particle Astrophysics and Cosmology, the Korean Scientist Group, the Chinese Academy of Sciences (LAMOST), Los Alamos National Laboratory, the Max-Planck-Institute for Astronomy (MPIA), the MaxPlanck-Institute for Astrophysics (MPA), New Mexico State University, Ohio State University, University of Pittsburgh, University of Portsmouth, Princeton University, the United States Naval Observatory, and the University of Washington. 


\section{REFERENCES}

Abazajian, K. N., Adelman-McCarthy, J. K., Agüeros, M. A., et al. 2009, ApJS, 182, 543

Aguerri, J. A. L., \& González-García, A. C. 2009, A\&A, 494, 891

Andersen, V. 1996, AJ, 111, 1805

Athanassoula, E. 2002, ApJ, 569, L83

-. 2003, MNRAS, 341, 1179

-. 2005, MNRAS, 358, 1477

Athanassoula, E., Machado, R. E. G., \& Rodionov, S. A. 2013, MNRAS, 429, 1949

Barazza, F. D., Jogee, S., \& Marinova, I. 2008, ApJ, 675, 1194

Barazza, F. D., Jablonka, P., Desai, V., et al. 2009, A\&A, 497, 713

Barrow, J. D., Bhavsar, S. P., \& Sonoda, D. H. 1984, MNRAS, $210,19 \mathrm{P}$

Barway, S., Wadadekar, Y., \& Kembhavi, A. K. 2011, MNRAS, 410, L18

Barway, S., Wadadekar, Y., Kembhavi, A. K., \& Mayya, Y. D. 2009, MNRAS, 394, 1991

Blanton, M. R., Schlegel, D. J., Strauss, M. A., et al. 2005, AJ, 129,2562

Boselli, A., \& Gavazzi, G. 2006, PASP, 118, 517

Brinchmann, J., Charlot, S., White, S.' D. M., et al. 2004, MNRAS, 351, 1151

Byrd, G., \& Valtonen, M. 1990, ApJ, 350, 89

Cameron, E., Carollo, C. M., Oesch, P., Aller, M. C., \& et al. 2010, MNRAS, 409, 346

Casteels, K. R. V., Bamford, S. P., Skibba, R. A., et al. 2013 , MNRAS, 429, 1051

Cervantes Sodi, B., Li, C., \& Park, C. 2014, submitted (arXiv:1409.6385)

Cervantes-Sodi, B., Li, C., Park, C., \& Wang, L. 2013, ApJ, 775, 19

Cheung, E., Athanassoula, E., Masters, K. L., et al. 2013, ApJ, 779,162

Choi, Y.-Y., Han, D.-H., \& Kim, S. S. 2010, Journal of Korean Astronomical Society, 43, 191

Christodoulou, D. M., Shlosman, I., \& Tohline, J. E. 1995, ApJ, 443,551

Coelho, P., \& Gadotti, D. A. 2011, ApJ, 743, L13

de Vaucouleurs, G. 1963, ApJS, 8, 31

de Vaucouleurs, G., de Vaucouleurs, A., Corwin, Jr., H. G., et al. 1991, S\&T, 82, 621

Debattista, V. P., \& Sellwood, J. A. 2000, ApJ, 543, 704

DeBuhr, J., Ma, C.-P., \& White, S. D. M. 2012, MNRAS, 426, 983

Efstathiou, G., Lake, G., \& Negroponte, J. 1982, MNRAS, 199, 1069

Elmegreen, B. G., \& Elmegreen, D. M. 1985, ApJ, 288, 438

Elmegreen, D. M., Elmegreen, B. G., \& Bellin, A. D. 1990, ApJ, 364,415

Erwin, P. 2005, MNRAS, 364, 283

Eskridge, P. B., Frogel, J. A., Pogge, R. W., et al. 2000, AJ, 119 536

Friedli, D., \& Benz, W. 1993, A\&A, 268, 65

Gerin, M., Combes, F., \& Athanassoula, E. 1990, A\&A, 230, 37

Giordano, L., Tran, K.-V. H., Moore, B., \& Saintonge, A. 2011, ArXiv e-prints

Giuricin, G., Mardirossian, F., Mezzetti, M., \& Monaco, P. 1993, ApJ, 407, 22

Heller, C. H., Shlosman, I., \& Athanassoula, E. 2007, ApJ, 671, 226

Hohl, F. 1971, ApJ, 168, 343

Hoyle, B., Masters, K. L., Nichol, R. C., et al. 2011, MNRAS, 415,3627

Hubble, E. P. 1936, Realm of the Nebulae

Jasche, J., Kitaura, F. S., Li, C., \& Enßlin, T. A. 2010, MNRAS, 409,355

Kauffmann, G., Heckman, T. M., White, S. D. M., et al. 2003 MNRAS, 341, 33

Knapen, J. H., Shlosman, I., \& Peletier, R. F. 2000, ApJ, 529, 93

Kormendy, J., \& Kennicutt, Jr., R. C. 2004, ARA\&A, 42, 603

Lansbury, G. B., Lucey, J. R., \& Smith, R. J. 2014, MNRAS, 439, 1749

Laurikainen, E., Salo, H., Buta, R., \& Knapen, J. H. 2009, ApJ, 692, L34
Lee, G.-H., Park, C., Lee, M. G., \& Choi, Y.-Y. 2012a, ApJ, 745, 125

Lee, G.-H., Woo, J.-H., Lee, M. G., et al. 2012b, ApJ, 750, 141

Li, C., Gadotti, D. A., Mao, S., \& Kauffmann, G. 2009, MNRAS, 397,726

Li, C., Kauffmann, G., Heckman, T. M., Jing, Y. P., \& White, S. D. M. 2008a, MNRAS, 385, 1903

Li, C., Kauffmann, G., Heckman, T. M., White, S. D. M., \& Jing, Y. P. 2008b, MNRAS, 385, 1915

Li, C., Kauffmann, G., Jing, Y. P., et al. 2006a, MNRAS, 368, 21

Li, C., Kauffmann, G., Wang, L., et al. 2006b, MNRAS, 373, 457

Long, S., Shlosman, I., \& Heller, C. 2014, ApJ, 783, L18

Marinova, I., \& Jogee, S. 2007, ApJ, 659, 1176

Marinova, I., Jogee, S., Heiderman, A., et al. 2009, ApJ, 698, 1639

Marinova, I., Jogee, S., Weinzirl, T., et al. 2012, ApJ, 746, 136

Martinez-Valpuesta, I., Shlosman, I., \& Heller, C. 2006, ApJ, 637, 214

Masters, K. L., Nichol, R. C., Hoyle, B., et al. 2011, MNRAS, 411,2026

Masters, K. L., Nichol, R. C., Haynes, M. P., et al. 2012, MNRAS, 424,2180

Méndez-Abreu, J., Sánchez-Janssen, R., \& Aguerri, J. A. L. 2010, ApJ, 711, L61

Méndez-Abreu, J., Sánchez-Janssen, R., Aguerri, J. A. L., Corsini, E. M. \& Zarattini, S. 2012, ApJ, 761, L6

Méndez-Hernández, H., Magaña, A. M., Hernández-Toledo, H. M., \& Valenzuela, O. 2011, in Revista Mexicana de Astronomia y Astrofisica Conference Series, Vol. 40, 78-79

Menéndez-Delmestre, K., Sheth, K., Schinnerer, E., Jarrett, T. H., \& Scoville, N. Z. 2007, ApJ, 657, 790

Miwa, T., \& Noguchi, M. 1998, ApJ, 499, 149

Mulchaey, J. S., \& Regan, M. W. 1997, ApJ, 482, L135

Nair, P. B., \& Abraham, R. G. 2010, ApJ, 714, L260

Noguchi, M. 1987, MNRAS, 228, 635

1988, A\&A, 203, 259

Oh, S., Oh, K., \& Yi, S. K. 2012, ApJS, 198, 4

Ostriker, J. P., \& Peebles, P. J. E. 1973, ApJ, 186, 467

Park, C., \& Choi, Y.-Y. 2005, ApJ, 635, L29

Romano-Díaz, E., Shlosman, I., Heller, C., \& Hoffman, Y. 2008, ApJ, 687, L13

Sakamoto, K., Okumura, S. K., Ishizuki, S., \& Scoville, N. Z. 1999, ApJ, 525, 691

Sellwood, J. 1980, The Messenger, 21, 27

Sellwood, J. A., \& Wilkinson, A. 1993, Reports on Progress in Physics, 56, 173

Sheth, K., Vogel, S. N., Regan, M. W., Thornley, M. D., \&

Teuben, P. J. 2005, ApJ, 632, 217

Sheth, K., Elmegreen, D. M., Elmegreen, B. G., et al. 2008, ApJ, 675,1141

Shlosman, I., Frank, J., \& Begelman, M. C. 1989, Nature, 338, 45

Skibba, R. A., Masters, K. L., Nichol, R. C., et al. 2012, MNRAS, 423,1485

Sundin, M., Donner, K. J., \& Sundelius, B. 1993, A\&A, 280, 105

Sundin, M., \& Sundelius, B. 1991, A\&A, 245, L5

Thompson, L. A. 1981, ApJ, 244, L43

Tremaine, S., \& Weinberg, M. D. 1984, MNRAS, 209, 729

van den Bergh, S. 2002, AJ, 124, 782

Wang, J., Kauffmann, G., Overzier, R., et al. 2012, MNRAS, 423, 3486

Weinberg, M. D. 1985, MNRAS, 213, 451

Weinzirl, T., Jogee, S., Khochfar, S., Burkert, A., \& Kormendy, J. 2009, ApJ, 696, 411

Whyte, L. F., Abraham, R. G., Merrifield, M. R., et al. 2002, MNRAS, 336, 1281

Yang, X., Mo, H. J., van den Bosch, F. C., et al. 2007, ApJ, 671, 153

-. 2005, MNRAS, 362, 711

Zehavi, I., Zheng, Z., Weinberg, D. H., et al. 2011, ApJ, 736, 59 